\title{
Mitral valve replacement for inflow obstruction of left ventricular assist device in a child with restrictive cardiomyopathy
}

\author{
Hari Tunuguntla, MD, ${ }^{a}$ Susan W. Denfield, MD, ${ }^{a}$ E. Dean McKenzie, MD, ${ }^{b}$ and Iki Adachi, MD, ${ }^{b}$ Houston, Tex
}

\footnotetext{
From the ${ }^{\mathrm{a} D e p a r t m e n t}$ of Pediatric Cardiology, Baylor College of Medicine, Houston, Tex; and the ${ }^{\mathrm{b}}$ Department of Congenital Heart Surgery, Texas Children's Hospital, Houston, Tex.

Disclosures: Authors have nothing to disclose with regard to commercial support.

Received for publication May 12, 2015; revisions received Aug 2, 2015; accepted for publication Aug 11, 2015; available ahead of print Oct 6, 2015.

Address for reprints: Iki Adachi, MD, Department of Congenital Heart Surgery, Baylor College of Medicine, 6621 Fannin St, Houston TX 77030 (E-mail: iadachi@bcm.edu).

J Thorac Cardiovasc Surg 2016;151:e11-3

$0022-5223 / \$ 36.00$

Copyright (C) 2016 by The American Association for Thoracic Surgery

http://dx.doi.org/10.1016/j.jtcvs.2015.08.031
}

Restrictive cardiomyopathy (RCM) poses a significant challenge in ventricular assist device (VAD) support. An associated stiff and small left ventricular (LV) cavity can result in obstruction to VAD inflow. ${ }^{1}$

\section{CLINICAL SUMMARY}

A 2-year-old male patient weighing $10.9 \mathrm{~kg}$ was admitted with a new diagnosis of RCM. The echocardiogram showed severely dilated atria and venae cavae (Figure 1, A). Cardiogenic shock developed, requiring emergency peripheral cannulation for extracorporeal membrane oxygenation. Development of pulmonary edema prompted conversion to central cannulation with the addition of a left atrial (LA) vent. After approval for a heart transplant, the patient was bridged to a Berlin Heart EXCOR LVAD (25-mL pump; Berlin Heart, The Woodland, Tex). Intraoperatively, the small LV barely accommodated a 9-mm LV inflow cannula. Cardiopulmonary bypass was

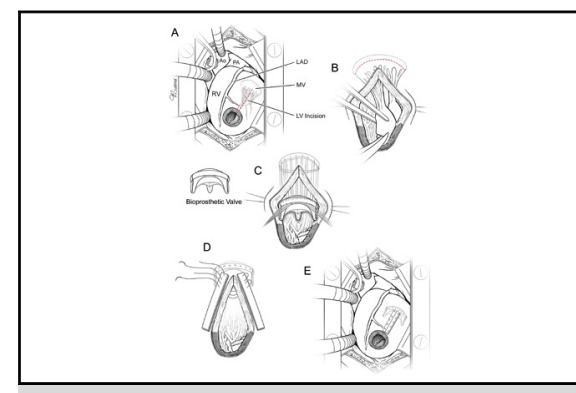

Approach to mitral valve replacement to enlarge LV cavity with LVAD placement. Printed with permission of Texas Children's Hospital.

\section{Central Message}

A novel surgical approach to left ventricular assist device management was used in a child with restrictive cardiomyopathy.

See Editorial Commentary page e15.

weaned with epinephrine and inhaled nitric oxide. Intraoperative transesophageal echocardiography showed a small LV cavity, with hypertrophied papillary muscles encroaching on the inflow cannula (Figure $1, B$ ). The sternum was closed with preserved pump filling. Overnight, severe inflow cannula obstruction developed, and he was taken back to the operating room. After establishment of

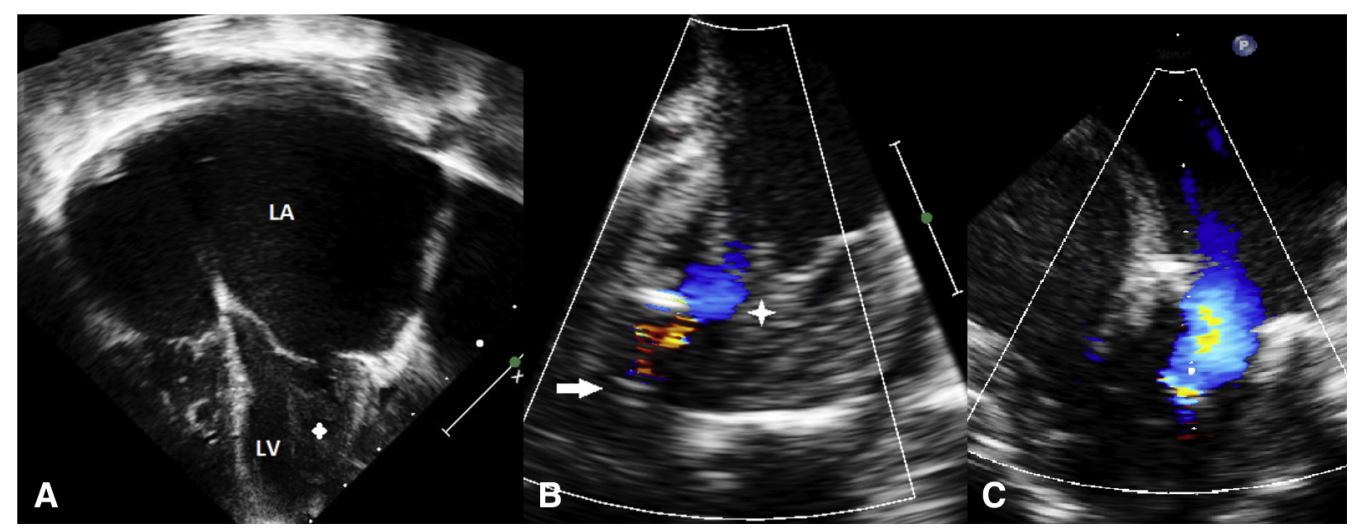

FIGURE 1. A, Transthoracic echocardiogram in the apical 4-chamber view showing a severely dilated left atrium, diminished left ventricular ( $L V$ ) cavity size, and hypertrophied papillary muscles (denoted by asterisk). B, Intraoperative transesophageal echocardiogram with left ventricular assist device placement. Cannula (denoted by arrow) is seen in the left ventricular cavity. Mitral valve and papillary muscle (denoted by asterisk) are encroaching on the inflow cannula in diastole. C, Intraoperative transesophageal echocardiogram after papillary muscle resection and prosthetic mitral valve replacement demonstrating laminar flow through the mitral valve into the left ventricular inflow cannula. $L A$, Left atrium. 

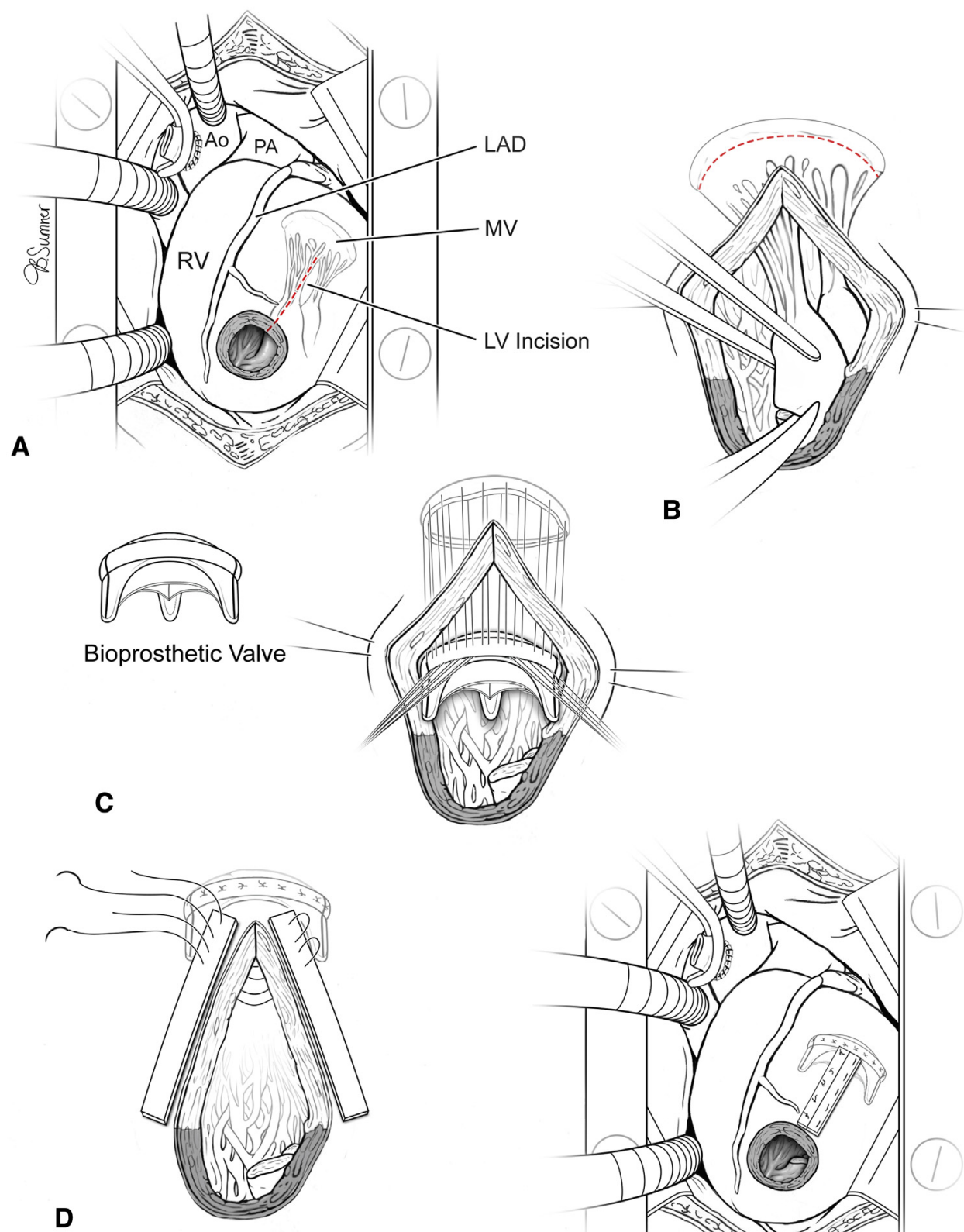

B
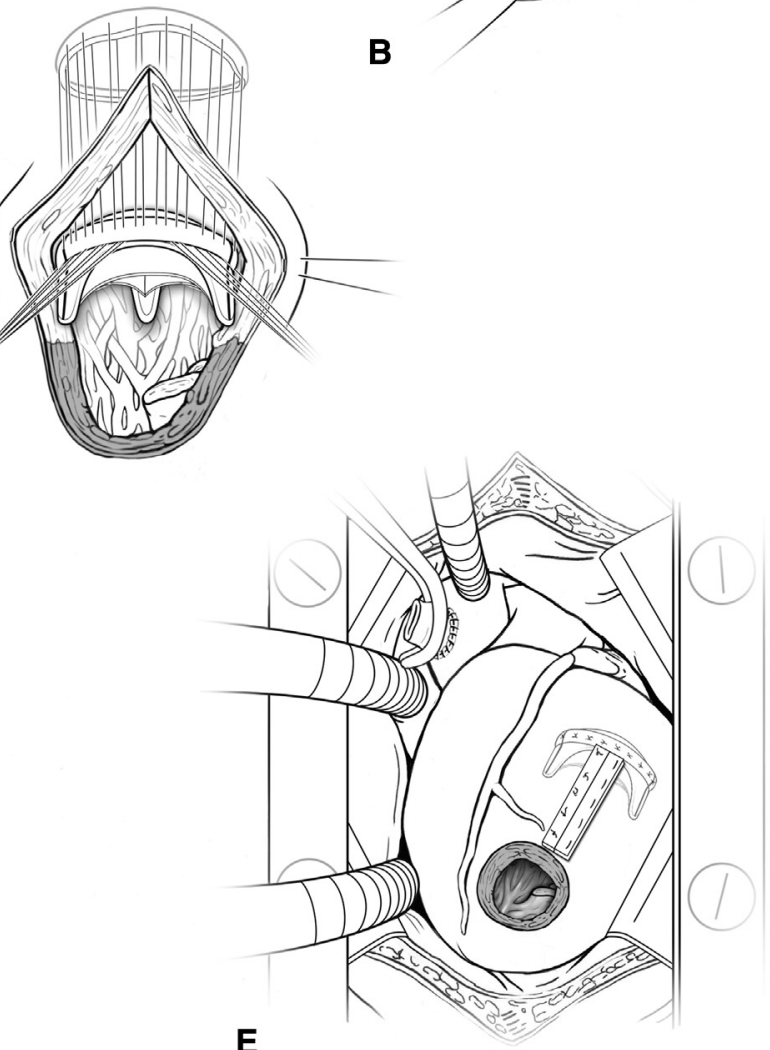

FIGURE 2. A, After establishment of cardiopulmonary bypass, the Berlin inflow and outflow cannulas are detached. The left ventricular $(L V)$ incision is indicated with a red dotted line. B and $\mathrm{C}$, The mitral valve $(M V)$ and its apparatus are excised, and a bioprosthetic valve is placed. D and $\mathrm{E}$, The ventriculotomy is reapproximated with interrupted horizontal mattress sutures over felt strips, leaving an opening for reinsertion of the Berlin inflow cannula. Ao, Aorta; $P A$, pulmonary artery; $L A D$, left anterior descending coronary artery; $R V$, right ventricle. Printed with permission of Texas Children's Hospital.

cardiopulmonary bypass, cardioplegia was given to arrest the heart, and the LV inflow cannula was detached from the LV apex. The resultant apical opening was extended toward the mitral valve parallel to the left anterior descending coronary artery (Figure 2). Through the created ventriculotomy, the hypertrophied papillary muscles and mitral valve apparatus were excised. A 19-mm porcine bioprosthetic valve (Epic; St Jude Medical, St Paul, Minn) was implanted with interrupted horizontal mattress pledgeted stitches. The ventriculotomy was reapproximated with interrupted horizontal mattress sutures over felt strips, leaving an opening for inflow cannula insertion 
at the apex. Left VAD support was reinstituted, with much improved filling of the pump. Echocardiography showed laminar inflow into the LV apical cannula with good function of the prosthetic mitral valve (Figure 1,C). Two weeks later, a large pericardial effusion developed, manifesting as poor left VAD filling. We then placed a right VAD to exclude right heart failure as a potential factor contributing to compromised filling. The patient was successfully bridged to orthotopic heart transplant after 5 months of biventricular assist device support without any thromboembolic or bleeding events. Anticoagulation was according to our standard protocol for the Berlin EXCOR. Inspection of the explanted heart revealed a wellpositioned mitral bioprosthetic valve without any thrombus formation.

\section{DISCUSSION}

Impediments to adequate LV filling in patients with a left VAD include a small LV cavity, obstruction to mitral valve inflow, and transient or persistent pulmonary hypertension. Children with RCM often have a small LV cavity size and pulmonary hypertension that can limit LV filling, resulting in VAD inflow cannula obstruction. ${ }^{1}$ In this patient, hypertrophied papillary muscles compounded the problem of a small LV cavity, resulting in an obstructive mitral valve apparatus and cannula obstruction with "suck-down" events.

To our knowledge, replacement of the mitral valve to address left VAD inflow obstruction has not been previously described. Mitral valvectomy alone may be considered sufficient to enlarge the LV cavity. We believe, however, that competency of the mitral valve is critical to avoid pulmonary edema, particularly with a pulsatile VAD, where the LV is not decompressed when the VAD is in systole (typically $40 \%$ of each pump cardiac cycle). Although the presence of a prosthetic mitral valve could potentially increase the risk of thromboembolic complications, data from a high-volume adult center suggest this was only a theoretic concern. ${ }^{2}$ Our patient did not have any hematologic complications with a standard anticoagulation regimen.

We recognize that this was an aggressive approach relative to an alternative option of LA cannulation. Although VAD support with LA cannulation was associated with a worse outcome, primarily as a result of thrombotic complications, in the previous adult experience, ${ }^{3}$ this may not be the case in children with RCM. According to the Berlin Heart registry as of 2011, there have been 30 EXCOR implantations worldwide in children with RCM, $40 \%(\mathrm{n}=12)$ with LA cannulation and the remaining $60 \%$ with LV cannulation. Mortality in the LV cannulation group was high at $39 \%$ (7 of 18). In contrast, there was a lower mortality in the LA cannulation group at $17 \%$ (2 of 12). This finding undoubtedly highlights the difficulty of VAD support in this group of patients. On the basis of this limited data set, even though it is orally reported to Berlin Heart's clinical affairs team and not audited, it may be reasonable to state that LA cannulation may be a safer approach in children with RCM relative to standard LV cannulation. With novel modifications of the LV cannulation technique, however, such as our method, the outcome of LV cannulation in RCM may become more favorable.

\section{References}

1. Topilsky Y, Pereira NL, Shah DK, Boilson B, Schirger JA, Kushwaha SS, et al, Left ventricular assist device therapy in patients with restrictive and hypertrophic cardiomyopathy. Circ Heart Fail. 2011;4:266-75.

2. Goda A, Takayama H, Koeckert M, Pak SW, Sutton EM, Cohen S, et al. Use of ventricular assist devices in patients with mitral valve prostheses. J Card Surg. 2011;26:334-7.

3. Holman WL, Bourge RC, Murrah CP, McGiffin DC, Spruell RD, Ferguson ER, et al. Left atrial or left ventricular cannulation beyond 30 days for a Thoratec ventricular assist device. ASAIO J. 1995;41:M517-22. 